\title{
Survey of Reactive Routing Protocols for MANET
}

\author{
Chintan Patel ${ }^{1}$, Prof. Vyomal N. Pandya ${ }^{2}$, Prof. Milind Shah ${ }^{3}$, \\ ${ }^{1}$ (Department of E.C. Engineering, Government Engineering College, Surat, India) \\ ${ }^{2}$ (Department of E.C. Engineering, C.K.P.C.E.T, Surat, India) \\ ${ }_{3}^{3}$ (Department of E.C. Engineering, Government Engineering College, Surat, India)
}

\begin{abstract}
A MANET is a collection of mobile nodes by wireless links forming a dynamic topology without any network infrastructure such as routers, servers, access points/cables or centralized administration. The nodes are free to move about and organize themselves into a network. These nodes change position frequently. The main classes of routing protocols are Proactive, Reactive and Hybrid. A Reactive (on-demand) routing strategy is a popular routing category for wireless ad hoc routing. It is a relatively new routing philosophy that provides a scalable solution to relatively large network topologies. The design follows the idea that each node tries to reduce routing overhead by sending routing packets whenever a communication is requested. DSR and AODV are reactive route discovery algorithms where a mobile device of MANET connects by gateway only when it is needed. The performance differentials are analyzed using varying performance metrics. These simulations are carried out using the ns-2 network simulator.
\end{abstract}

Keywords - AODV, DSR, MANET, NS-2, Reactive routing protocols, Survey.

\section{Introduction}

The wireless networks are classified as Infrastructured or Infrastructure less. In Infrastructured wireless networks, the mobile node can move while communicating, the base stations are fixed and as the node goes out of the range of a base station, it gets into the range of another base station. In Infrastructure less or Ad Hoc wireless network, the mobile node can move while communicating, there are no fixed base stations and all the nodes in the network act as routers. The mobile nodes in the Ad Hoc network dynamically establish routing among themselves to form their own network 'on the fly'.

In areas where there is little [2] or no communication infrastructure or the existing infrastructure is expensive or inconvenient to use, wireless mobile users may be able to communicate through the formation of an Ad-hoc Network. In such a network, each mobile node does not operate only as a host but also as a router, forwarding packets for other mobile nodes in the network that may not be within direct wireless transmission range of each other. Each node participates in an ad hoc routing protocol that allows it to discover "multi-hop" paths through the network to any other node. The idea of ad hoc networking is sometimes also called infrastructure less networking. Some examples of the possible uses of ad hoc networking include, students using laptop computers to participate in an interactive lecture, business associates sharing information during a meeting, soldiers relaying information for situational awareness on the battlefield, and emergency disaster relief personnel coordinating efforts after a hurricane or earthquake.

\subsection{MANET (Mobile Ad-hoc NETwork)}

The MANET [2] is a collection of nodes, which have the possibility to connect on a wireless medium and form an arbitrary and dynamic network with wireless links. This means that links between the nodes can change with time, new nodes can join the network, and other nodes can leave it. A MANET is expected to be of larger size than the radio range of the wireless antennas, because of this fact it could be necessary to route the traffic through a multi-hop path to give two nodes the ability to communicate. There are neither fixed routers nor fixed locations for the routers as in cellular networks - also known as infrastructure networks.

Cellular networks consist of a wired backbone, which connects the base-stations. The mobile nodes can only communicate over a one-hop wireless link to the base-station; multi-hop wireless links are not possible. By contrast, a MANET has no permanent infrastructure at all. All mobile nodes act as mobile routers. A MANET [2] is highly dynamic. Links and participants are often changing and the quality of the links as well. Furthermore, asymmetric links are also possible. New routing protocols are needed to satisfy the specific requirements of mobile Ad hoc networks. There exists a large family of ad hoc routing protocols. 


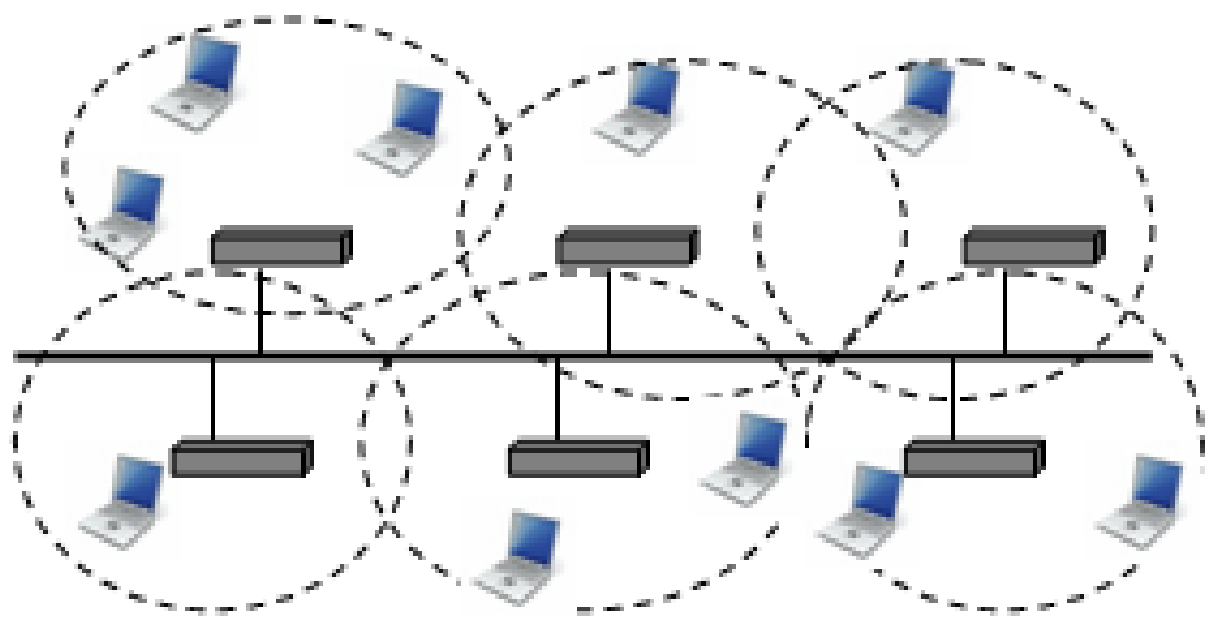

Fig. 1 Wireless Network Structures (Infrastructure Networks) [2]

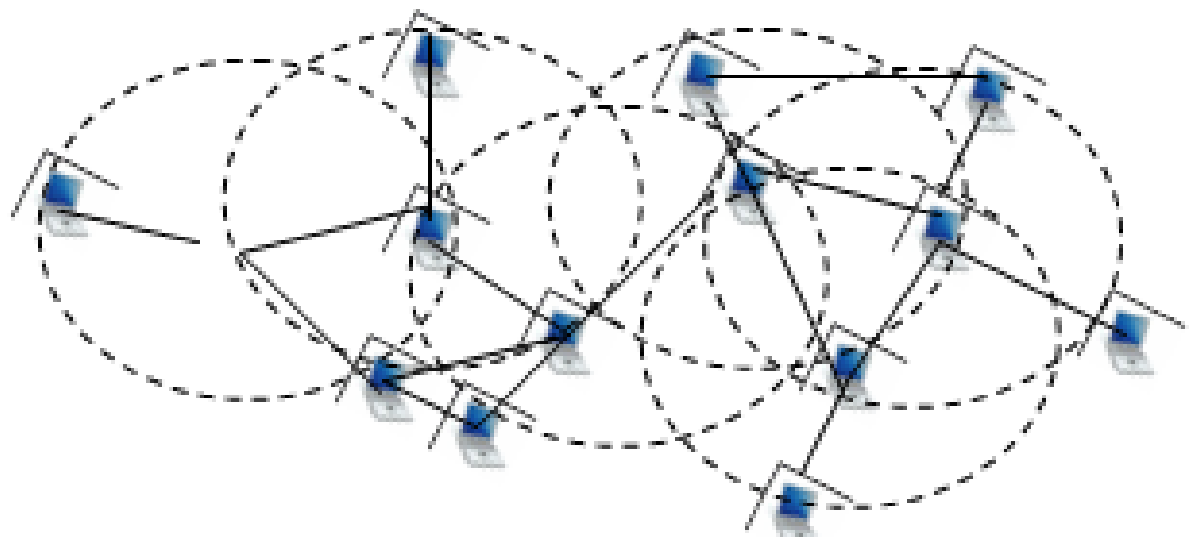

Fig.2 Wireless Network Structures (Infrastructure less Networks) [2]

Mobile Ad-hoc Networks (MANETs) [5] are autonomous self-organized networks without the aid of any established infrastructure or centralized administration (e.g., base stations or access points). Communication is done through wireless links among mobile hosts through their antennas. Due to concerns such as radio power limitation and channel utilization, a mobile host may not be able to communicate directly with other hosts in a single hop fashion. In this case, a multi-hop scenario occurs, in which the packets sent by the source host must be relayed by several intermediate hosts before reaching the destination host. Thus, each mobile host in a MANET must function as a router to discover and maintain routes to other nodes in the network.

In a Mobile Ad-hoc Network [5], nodes move arbitrarily, therefore the network may experience rapid and unpredictable topology changes. The topology of the mobile ad-hoc network depends on the transmission power of the nodes and the location of the mobile nodes, which may change with time. In general, Mobile Ad-hoc Networks are self-creating, self-organizing, and self-administrating networks.

Characteristics of MANET [7]

- Communication via wireless means.

- Nodes can perform the roles of both hosts and routers.

- Bandwidth-constrained, variable capacity links.

- Energy-constrained Operation.

- $\quad$ Limited Physical Security.

- Dynamic network topology.

- Frequent routing updates.

Advantages of MANET [7]

- They provide access to information and services regardless of geographic position.

- These networks can be set up at any place and time.

Disadvantages of MANET [7]

- Limited resources and physical security.

- Intrinsic mutual trust vulnerable to attacks.

- Lack of authorization facilities.

- Volatile network topology makes it hard to detect malicious nodes. 
- Security protocols for wired networks cannot work for ad hoc networks.

APPLICATIONS OF MANET [7]

- Military or police exercises.

- Disaster relief operations.

- Mine cite operations.

- Urgent Business meetings.

\section{Routing Protocols In Manet}

Classification [6] of Routing Protocols for Ad Hoc Wireless Networks:

(1) Based on Routing information update mechanism

- Table Driven(Proactive)

$$
>\text { DSDV, WRP, CGSR, STAR, etc. }
$$

- On-Demand(Reactive)

- Hybrid

$>$ AODV, DSR, SSA, ABR, etc.

(2) Based on the use of temporal information

- Path Selection using Past History

$>$ DSDV, WRP, STAR, AODV, DSR, etc.

- $\quad$ Path Selection using Prediction

$>$ LBR, RABR, etc.

(3) Based on topology information

- Flat Routing

$>$ AODV, DSR, SSA, ABR, etc.

- Hierarchical Routing

$>$ CGSR.

(4) Based on utilization of specific resources

- $\quad$ Power Aware Routing

$>$ PAR

- Routing Using Geographical Information

$>$ LAR

- $\quad$ Routing with Efficient Flooding

$>$ Table Driven(OLSR)

$>$ On-Demand(PLBR)

A routing protocol [1] is needed whenever a packet needs to be transmitted to a destination via number of nodes and numerous routing protocols have been proposed for such kind of ad hoc networks. These protocols find a route for packet delivery and deliver the packet to the correct destination. The studies on various aspects of routing protocols have been an active area of research for many years. Many protocols have been suggested keeping applications and type of network in view. Basically, routing protocols can be broadly classified into two types as: Table Driven Protocols or Proactive Protocols and On-Demand Protocols or Reactive Protocols. In Table Driven routing protocols each node maintains one or more tables containing routing information to every other node in the network. All nodes keep on updating these tables to maintain latest view of the network. Some of the existing table driven protocols are DSDV, GSR, WRP and ZRP. In on-demand routing protocols, routes are created as and when required. When a transmission occurs from source to destination, it invokes the route discovery procedure. The route remains valid till destination is achieved or until the route is no longer needed. Some of the existing on demand routing protocols are: DSR, AODV and TORA.

\subsection{Proactive/Table-Driven Routing Protocols}

In proactive routing protocols [2], each node maintains routing information to every other node (or nodes located in a specific part) in the network. The routing information is usually kept in a number of different tables. These tables are periodically updated and/or if the network topology changes. The difference between these protocols exists in the way the routing information is updated, and the type of information kept at each routing table. Keeping routes to all destinations up-to-date, even if they are not used, is a disadvantage with regard to the usage of bandwidth and of network resources. It is also possible that the control traffic delays data packets, because queues are filled with control packets and there are more packet collisions due to more network traffic. Proactive protocols do not scale in the frequency of topology change. Therefore the proactive strategy is appropriate for a low mobility network. 
Table driven routing protocol [3] attempt to maintain consistent, up-to-date routing information from each node to every other node in the network. These protocols require each node to maintain one or more tables to store routing information, and they respond to changes in network topology by propagating updates routes throughout the network in order to maintain a consistent network view. The Destination-Sequenced DistanceVector Routing (DSDV) protocol is a table driven algorithm that modifies the Bellman-Ford routing algorithm to include timestamps that prevent loop-formation. The Wireless Routing Protocol (WRP) is a distance vector routing protocol which belongs to the class of path-finding algorithms that exchange second-to-last hop to destinations in addition to distances to destinations. This extra information helps remove the "counting-toinfinity" problem that most distance vector routing algorithms suffer from. It also speeds up route convergence when a link failure occurs.

\subsection{Hybrid Routing Protocols}

Based on combination of both table and demand driven routing protocols, some hybrid routing protocols [3] are proposed to combine advantage of both proactive and reactive protocols. The most typical hybrid one is zone routing protocol (ZRP).

\subsection{Reactive/ On-Demand Routing Protocols}

These protocols [2] were designed to overcome the wasted effort in maintaining unused routes. Routing information is acquired only when there is a need for it. The needed routes are calculated on demand. This saves the overhead of maintaining unused routes at each node, but on the other hand the latency for sending data packets will considerably increase. It is obvious that a long delay can arise before data transmission because it has to wait until a route to the destination is acquired. As reactive routing protocols flood the network to discover the route, they are not optimal in terms of bandwidth utilization, but they scale well in the frequency of topology change. Thus this strategy is suitable for high mobility networks. Reactive protocols can be classified into two categories, Source routing and Hop-by-hop routing. In Source routed on-demand protocols, each data packets carry the complete source to destination address. Therefore, each intermediate node forwards these packets according to the information kept in the header of each packet. This means that the intermediate nodes do not need to maintain up-to-date routing information for each active route in order to forward the packet towards the destination. Furthermore, nodes do not need to maintain neighbor connectivity through periodic beaconing messages neighbors through the use of beaconing messages. In hop-by-hop routing (also known as point-to-point routing), each data occurs by coding route request packets through packet only carries the destination address and the next hop address. Therefore, each intermediate node in the path to the destination uses its routing table to forward each data packet towards the destination.

A different approach from table-driven routing [3] is on demand routing. This type of routing creates routes only when desired by source node. When a node requires a route to a destination, it initiates a route discovery process within the network. This process is completed once a route is found or all possible routes permutations have been examined. Once a route has been established, it is maintained by a route maintenance procedure until either the destination becomes inaccessible along every path from the source or until the route is no longer desired. On-demand routing protocols were designed with the aim of reducing control overhead, thus increasing bandwidth and conserving power at the mobile stations. These protocols limit the amount of bandwidth consumed by maintaining routes to only those destinations for which a source has data traffic. Therefore, the routing is source-initiated as opposed to table driven routing protocols that are destination initiated. There are several recent examples of this approach (e.g., AODV, DSR, TORA, ZRP) and the routing protocols differ on the specific mechanisms used to disseminate flood search packets and their responses, cache the information heard from other nodes' searches, determine the cost of a link, and determine the existence of a neighbour. However, all the on-demand routing proposals use flood search messages that either: (a) give sources the entire paths to destinations, which are then used in source routed data packets (e.g., DSR); or (b) provide only the distances and next hops to destinations, validating them with sequence numbers (e.g., AODV) or time stamps (e.g., TORA).

\subsubsection{Ad hoc On-demand Distance Vector (AODV)}

Ad hoc On-demand distance vector (AODV) [3] is another variant of classic distance vector routing algorithm, based on DSDV and DSR. It shares DSR on-demand characteristics, discovers routes on an as needed basis via a similar route discovery process. However, AODV adopts traditional routing tables; one entry per destination which is in contrast to DSR that preserves multiple route cache entries for each destination. The early design of AODV is undertaken after the experience with DSDV routing algorithm. Like DSDV, AODV provides loop free routes in case of link breakage but unlike DSDV, it doesn't need global periodic routing advertisement. AODV uses a broadcast route discovery algorithm and then the unicast route reply massage. The following sections explain these mechanisms in more details. 
Route Discovery

When a node wants to send a packet to some destination and does not have a valid route in its routing table for that destination, initiates a route discovery. Source node broadcasts a route request (RREQ) packet to its neighbours, which then forwards the request to their neighbours and so on shown in figure 2.3. To control network-wide broadcasts of RREQ packets, the source node use an expanding ring search technique. In this technique, source node starts searching the destination using some initial time to live (TTL) value. If no reply is received within the discovery period, TTL value incremented by an increment value. This process will continue until the threshold value is reached. When an intermediate node forwards the RREQ, it records the address of the neighbour from which first packet of the broadcast is received, thereby establishing a reverse path.

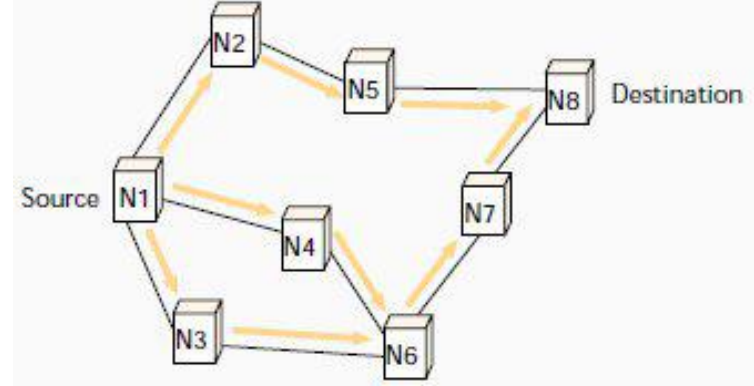

(a) Propagation of the RREQ

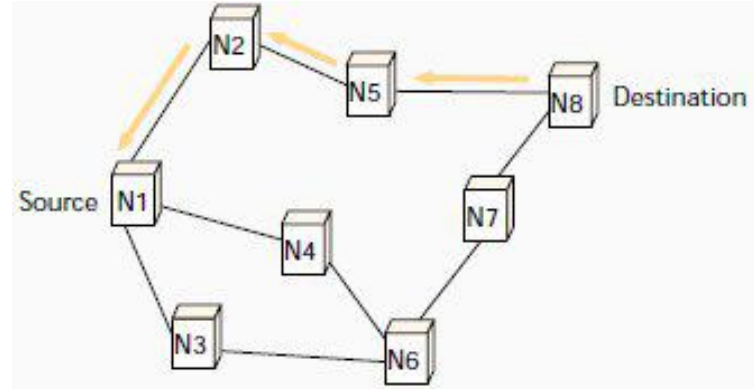

(b) Path of the RREP to the source

Fig. 3: AODV Path Discovery Process [3]

When the RREQ reaches a node that is either the destination node or an intermediate node with a fresh enough route to the destination, replies by unicasting the route reply (RREP) towards the source node. As the RREP is routed back along the reverse path shown figure 2.3, intermediate nodes along this path set up forward path entries to the destination in its route table and when the RREP reaches the source node, a route from source to the destination establish.

Route Maintenance

A route established between source and destination pair is maintained as long as needed by the source. If the source node moves during an active session, it can reinitiate route discovery to find out a new route to destination. However, if the destination or some intermediate node moves, the node upstream of the break remove the routing entry and send route error (RERR) message to the affected active upstream neighbours. These nodes in turn propagate the RERR to their precursor nodes, and so on until the source node is reached. The affected source node may then choose to either stop sending data or reinitiate route discovery for that destination by sending out a new RREQ message.

Advantages and Drawbacks of AODV

AODV protocol has number of advantages [1] too. The routes are established on demand and destination sequence numbers are used to find the latest route to the destination. The connection setup delay is lower. It also responds very quickly to the topological changes that affects the active routes. It does not put any additional overheads on data packets as it does not make use of source routing. It favours the least congested route instead of the shortest route and it also supports both unicast and multicast packet transmissions even for nodes in constant movement. AODV has also certain drawbacks [1] like DSR. The intermediate nodes can lead to inconsistent routes if the source sequence number is very old and the intermediate nodes have a higher but not the latest destination sequence number, thereby having stale entries. The various performance metrics begin decreasing as the network size grows. It is vulnerable to various kinds of attacks as it based on the assumption that all nodes must cooperate and without their cooperation no route can be established. The multiple Route Reply packets in response to a single Route Request packet can lead to heavy control overhead. The periodic beaconing leads to unnecessary bandwidth consumption. It expects/requires that the nodes in the broadcast medium can detect each others' broadcasts. It is also possible that a valid route is expired and the determination of a reasonable expiry time is difficult. The reason behind this is that the nodes are mobile and their sending rates may differ widely and can change dynamically from node to node.

\subsubsection{Dynamic Source Routing (DSR)}

The Dynamic Source Routing (DSR) is one of the purest examples of an on-demand routing protocol that is based on the idea of source routing. It is designed specially for use in multihop ad hoc networks for mobile nodes. It allows the network to be completely self-organizing and self-configuring and does not need any existing network infrastructure or administration. DSR [3] uses no periodic routing messages like AODV, thereby reduces network bandwidth overhead, conserves battery power and avoids large routing updates. Instead DSR needs support from the MAC layer to identify link failure. DSR is composed of the two mechanisms of 
Route Discovery and Route Maintenance, which work together to allow nodes to discover and maintain source routes to arbitrary destinations in the network show in figure 4 . The following sections explain these mechanisms in more details:

Route Discovery

When a mobile node has a packet to send to some destination shown figure 4, it first checks its route cache to decide whether it already has a route to the destination. If it has an unexpired route, it will use this route to send the packet to the destination. On the other hand, if the cache does not have such a route, it initiates route discovery by broadcasting a route request packet shown in figure 4 . Each node receiving the route request packet searches throughout its route cache for a route to the intended destination. If no route is found in the cache, it adds its own address to the route record of the packet and then forwards the packet to its neighbours.

This request propagates through the network until either the destination or an intermediate node with a route to destination is reached. Whenever route request reaches either to the destination itself or to an intermediate node which has a route to the destination, a route reply is unicasted back to its originator.

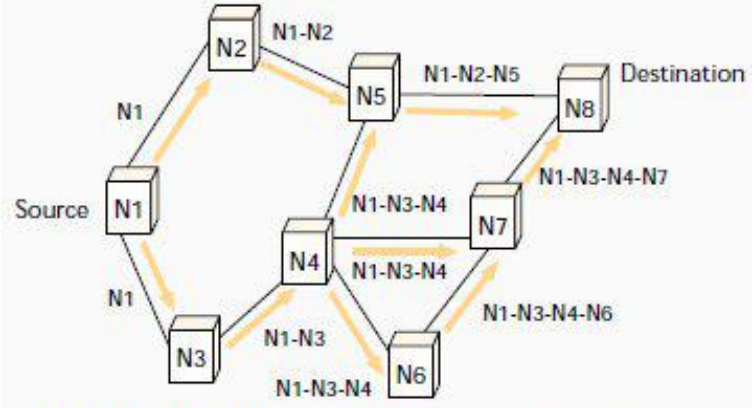

(a) Building of the route record during route discovery

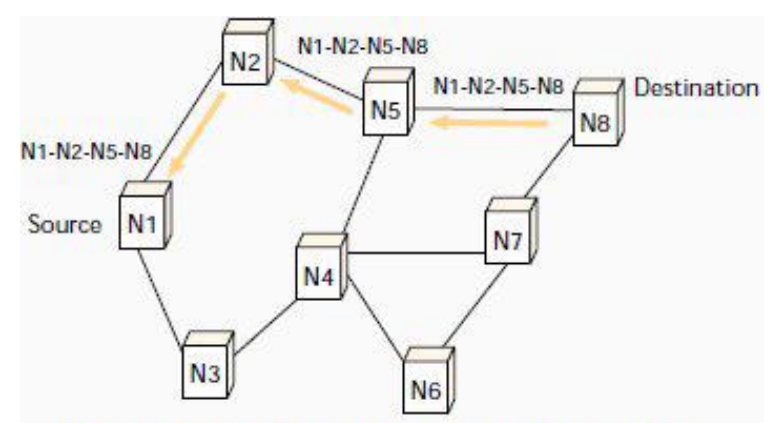

(b) Propagation of the route reply with the route record

Fig. 4: DSR Route Discovery Process [3]

Route Maintenance

In DSR, route is maintained by using route error packets and acknowledgments. When a packet with source route is originated or forwarded, each node sending the packet is responsible for confirming that the packet has been received by the next hop. The packet is retransmitted until the conformation of receipt is received. If the packet is transmitted by a node the maximum number of times and yet no receipt information is received, this node returns a route error message to the source of the packet. When this route error packet is received, the hop in error is removed from the host's route cache and all routes containing the hop are truncated at that point.

Advantages and Drawbacks of DSR

DSR protocol has number of advantages [1]. It does not use periodic routing messages (e.g. no router advertisements and no link-level neighbour status messages), thereby reducing network bandwidth overhead, conserving battery power, and avoiding the propagation of potentially large routing updates throughout the ad hoc network. There is no need to keep routing table so as to route a given data packet as the entire route is contained in the packet header. The routes are maintained only between nodes that need to communicate. This reduces overhead of route maintenance. Route caching can further reduce route discovery overhead. A single route discovery may yield many routes to the destination, due to intermediate nodes replying from local caches. The DSR protocol guarantees loop-free routing and very rapid recovery when routes in the network change. It is able to adapt quickly to changes such as host movement, yet requires no routing protocol overhead during periods in which no such changes occur. In addition, DSR has been designed to compute correct routes in the presence of asymmetric (uni-directional) links. In wireless networks, links may at times operate asymmetrically due to sources of interference, differing radio or antenna capabilities, or the intentional use of asymmetric communication technology such as satellites. Due to the existence of asymmetric links, traditional link-state or distance vector protocols may compute routes that do not work. DSR, however, will find a correct route even in the presence of asymmetric links.

DSR protocol is not totally free from drawbacks [1] as it is not scalable to large networks. It is mainly efficient for mobile ad hoc networks with less than two hundred nodes. DSR requires significantly more processing resources than most other protocols. In order to obtain the routing information, each node must spend lot of time to process any control data it receives, even if it is not the intended recipient. The contention is increased if too many route replies come back due to nodes replying using their local cache. The Route Reply Storm problem is there. An intermediate node may send Route Reply using a stale cached route, thus polluting other caches. This problem can be eased if some mechanism to purge (potentially) invalid cached routes is incorporated. 
The Route Maintenance protocol does not locally repair a broken link. The broken link is only communicated to the initiator. Packet header size grows with route length due to source routing. Flood of route requests may potentially reach all nodes in the network. Care must be taken to avoid collisions between route requests propagated by neighbouring nodes.

\section{Network Simulator-2}

Ns-2 [7] is a discrete event simulator targeted at networking research. It provides substantial support for simulation of TCP, routing and multicast protocols over wired and wireless networks. It consists of two simulation tools. The network simulator (ns) contains all commonly used IP protocols. The network animator (nam) is use to visualize the simulations. Ns-2 fully simulates a layered network from the physical radio transmission channel to high-level applications. Version 2 is the most recent version of ns (ns-2). The simulator was originally developed by the University of California at Berkeley and VINT project the simulator was recently extended to provide simulation support for ad hoc network by Carnegie Mellon University (CMU Monarch Project homepage, 1999). The ns-2 simulator has several features that make it suitable for our simulations.

A network environment for ad-hoc networks, Wireless channel modules (e.g.802.11), Routing along multiple paths, Mobile hosts for wireless cellular networks. Ns-2 is an objectoriented simulator written in C++ and OTcl. The simulator supports a class hierarchy in $\mathrm{C}++$ and a similar class hierarchy within the OTcl interpreter. There is a one-to-one correspondence between a class in the interpreted hierarchy and one in the compile hierarchy. The reason to use two different programming languages is that OTcl is suitable for the programs and configurations that demand frequent and fast change while $\mathrm{C}++$ is suitable for the programs that have high demand in speed. Ns-2 [7] is highly extensible. It not only supports most commonly used IP protocols but also allows the users to extend or implement their own protocols. It also provides powerful trace functionalities, which are very important in our project since various information need to be logged for analysis. The full source code of ns-2 can be downloaded and compiled for multiple platforms such as UNIX, Windows and Cygwin.

\section{Simulation Results}

For simulation and performance analysis of reactive routing protocols like AODV and DSR, we need to select one of the performance metrics from Throughput, Average Throughput, End-To-End Delay, Mobility speed of nodes, Pause time, etc. Here, Throughput is selected to analyze the performance by comparison of reactive routing protocols (AODV and DSR). It is defined as,

Throughput: It is the ratio of the total packets delivered to the destination to the time taken for deliver the packets.

Throughput $=($ total packets delivered to the destination $) /($ time taken for deliver the packets $)$

For simulation, some parameters should be defined which are selected as follow in this case

Table1: Simulation Parameters

\begin{tabular}{cc}
\hline Parameter & Value \\
\hline Simulation Time & $1500 \mathrm{sec}$ \\
Number of Nodes & 2 \\
Routing Protocols & AODV, DSR \\
Simulation Area & $500 \times 400 \operatorname{meter}^{\wedge} 2$ \\
Packet Size & 500 \\
Interval & 0.05 \\
\hline
\end{tabular}

The network animator for two nodes using TCP traffic is shown in the fig. 5. We can see that the node_0 work as source node and transmits traffic to node_1 while node_1 works as a destination and transmits acknowledgement to node_0. Fig. 6 shows the comparison of throughput for reactive routing protocols(AODV and DSR). The AODV gives better throughput compared to DSR because of least congested path. 


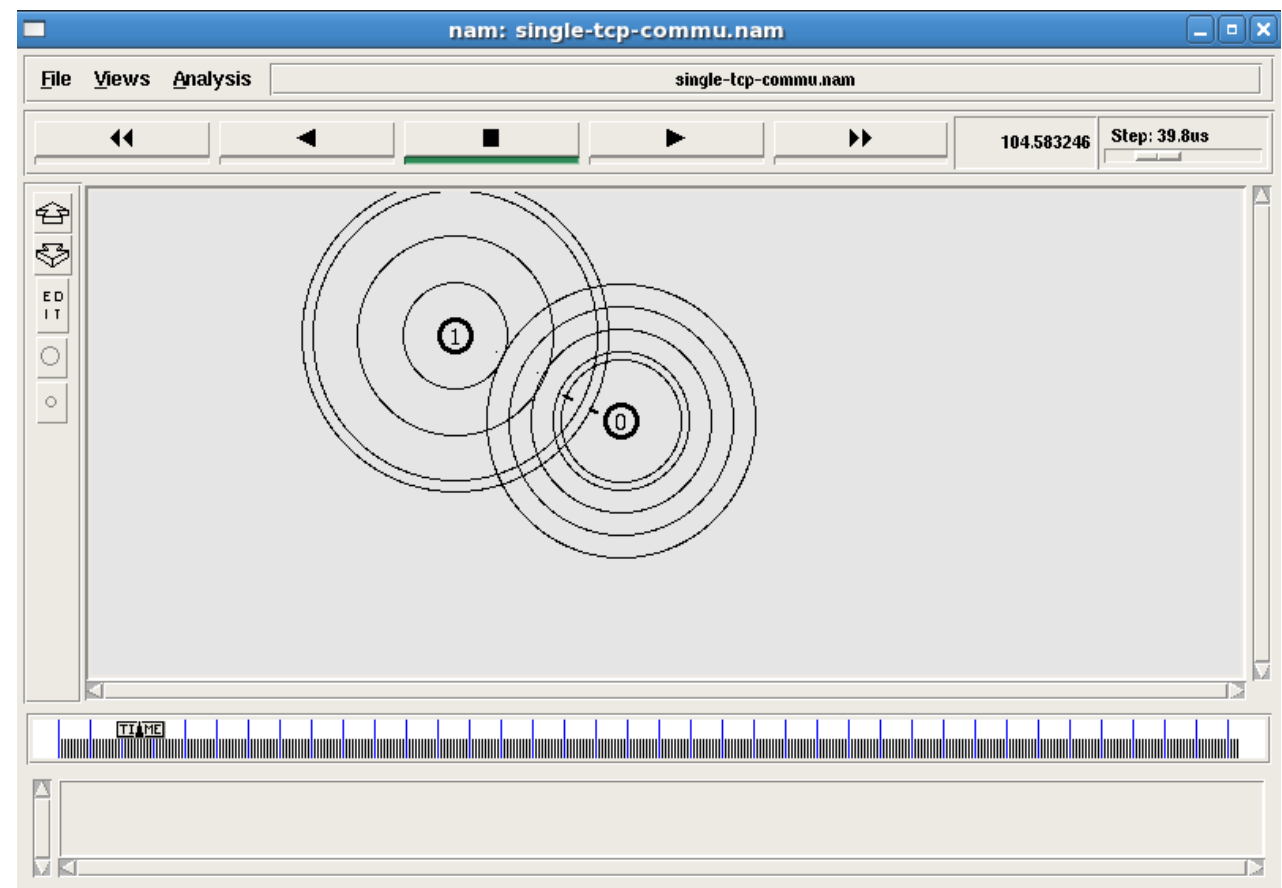

Fig. 5: Network Animator for Simulation using two nodes with TCP traffic

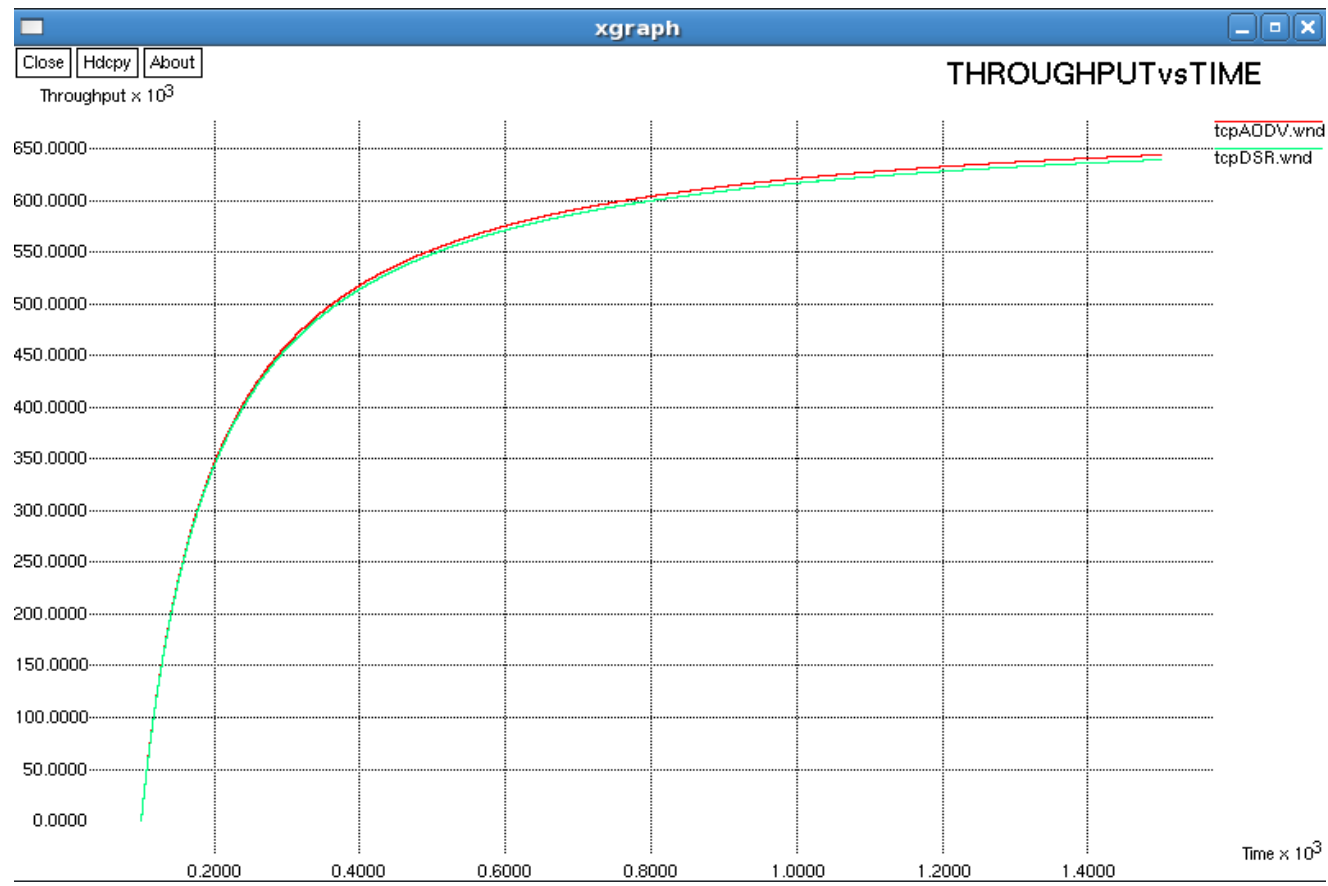

Fig. 6: Comparison of Throughput for reactive routing protocols AODV and DSR

\section{Conclusion}

By surveying about the reactive routing protocols, we come to know in detail about reactive routing protocols and observe that AODV selects least congested path for traffic flow with minimum possibility of packet drop while DSR selects shortest path with higher possibility of packet drop compared to AODV. We have compared two Reactive (On-demand) Routing Protocols, namely, Ad hoc On-Demand Distance Vector Routing (AODV) and Dynamic Source Routing (DSR). As the traffic parameter we have used File Transfer Protocol (FTP). We analyze both protocols in terms of Throughput. As the time increases, the Throughput increases in AODV and DSR. By comparison of throughput of both the routing protocols, we can observe that AODV gives higher throughput compared to DSR in wireless ad hoc network, which matches with our surveying results. 


\section{References}

[1] Sunil Taneja, Ashwani Kush and Amandeep Makkar, "Experimental Analysis of DSR, AODV using Speed and Pause time", International Journal of Innovation, Management and Technology, Vol. 1, No. 5, December 2010, 453-458, ISSN: $2010-0248$.

[2] Anil Kumar Sharma and Neha Bhatia, "Behavioral Study of MANET Routing Protocols by using NS-2", International Journal of Computational Engineering \& Management, Vol. 12, April 2011, 100-104, ISSN (Online): 2230-7893, www.IJCEM.org.

[3] Dhananjay Bisen, Preetam Suman, Prof. Sanjeev Sharma and Rajesh Shukla, "Effect of Pause Time on DSR, AODV and DYMO Routing Protocols in MANET", Manuscript received November 20, 2009.

[4] Manijeh Keshtgary and Vahide Babaiyan, "Performance Evaluation of Reactive, Proactive and Hybrid Routing Protocols in MANET”, Manijeh Keshtgary et al. / International Journal on Computer Science and Engineering (IJCSE), Vol. 4(2), Feb. 2012, 248-254, ISSN: 0975-3397.

[5] Baldev Ram Mali and N.C. Barwar, "Effect of Mobility on Performance of MANET Routing Protocols under Different Traffic Patterns", IP Multimedia Communications, A Special Issue from IJCA - www.ijcaonline.org, 19-24.

[6] C. Siva Ram Murthy and B. S. Manoj, “Ad Hoc Wireless Networks Architectures and Protocols”, Prentice Hall, PTR, 2004.

[7] Sachin Dnyandeo Ubarhande, "Performance Evolution of AODV and DSR Routing Protocols in MANET Using NS2", International Journal of Scientific and Engineering Research, Vol.3, Issue 5, May 2012, 1-5, ISSN: 2229-5518. 